\title{
Avaliação de Algoritmos Genéticos Puros e Híbridos para Index Tracking
}

\author{
Thiago Wanderley de Amorim ${ }^{1}$, Julio Cezar Soares Silva ${ }^{2}$ and Adiel Teixeira de Almeida Filho ${ }^{2}$ \\ ${ }^{1}$ Electrical Engineering Department, Universidade Federal de Pernambuco, Recife, Brazil \\ ${ }^{2}$ Centro de Informática, Universidade Federal de Pernambuco, Recife, Brazil \\ Emails: \{twa,jcss4,adielfilho\}@ cin.ufpe.br
}

\begin{abstract}
O problema de index tracking estuda a reprodução da performance de um índice com um portfólio formado por um número restrito de ativos. Esta abordagem é especialmente importante em situações reais, onde custos de transação estão associados à compra e venda de ativos, impossibilitando uma replicação fiel do benchmark. No entanto, essa restrição de cardinalidade do portfólio acrescenta uma complexidade adicional, por isso, sua solução pode levar muito tempo. Para contornar este problema, metaheurísticas foram propostas com o objetivo de alcançar boas soluções em um tempo razoável. Este trabalho analisa a performance de cinco algortimos genéticos aplicados a duas formulações de modelos para a otimização deste problema de seleção portfólio: um linear e outro quadrático, em termos de tempo de execução e qualidade da solução. Os resultados mostram que a abordagem híbrida dos GAs foi capaz de retornar soluções competitivas em menos tempo do que um solver genérico, tanto em períodos de otimização simples quanto em estratégias de rebalanceamento do portfólio, de forma a ser uma alternativa confiável à um gestor de fundos para ajudar em seu processo de tomada de decisão, permitindo diversos experimentos em um curto período de tempo antes da formação do portfólio.

Index Terms-Index tracking, Seleção de portfólio, Algoritmo genético, backtesting
\end{abstract}

\section{INTRODUÇÃO}

O problema de index tracking consiste em replicar, da forma mais próxima possível, a performance de um índice. Dada esta situação, um gestor de ativos pode adotar duas abordagens: replicação completa ou parcial do índice. Para a replicação completa, cabe ao gestor comprar todos os ativos presentes no índice em suas devidas proporções, de forma a mimetizar perfeitamente o benchmark. Na prática esta abordagem não é realista, visto que acarreta altos custos de transação.

Resta então a segunda abordagem: montar uma carteira com uma quantidade menor de ativos, mas que possua a performance mais próxima possível do índice em questão. De forma a auxiliar esta tomada de decisão, diversos modelos de otimização foram propostos, com a adição de restrições que buscam aproximar o modelo de situações reais, na tentativa de representar melhor a realidade e melhorar a performance da solução.

O objetivo em um modelo de otimização é aproximar a performance do portfólio de solução (tracking portfolio) da performance do benchmark, isto é, minimizar a diferença entre as duas performances. Esta medida é chamada de tracking error. A literatura propõe diversas definições para o tracking error [1]-[4]. Neste trabalho, foram usados dois modelos, um com definição linear do tracking error, e outro com definição quadrática.

Apesar de aproxima-lo da realidade, a adição de restrições ao modelo aumenta sua complexidade computacional. A restrição da quantidade de ativos que compõem o tracking portfolio está na natureza de um problema de index tracking, tendo em vista a necessidade de reduzir os ativos que fazem parte da solução. Essa restrição leva o nome de restrição de cardinalidade, e sua natureza combinatória torna o problema NP-Hard [2]. Portanto, sua solução ótima pode levar horas ou dias para ser obtida, a medida que o tamanho do universo de ações analisado aumenta, tempo impraticável em uma situação real.

A fim de obter-se boas soluções em tempos razoáveis, foram propostas diversas heurísticas [5]-[7]. Estas abordagens podem ser úteis em momentos de rebalanceamento de portfólio e mudanças no cenário, possibilitando ao gestor simular diversas situações e fazer testes em tempo oportuno, de modo a ajudar em sua tomada de decisão [5], [8].

Este trabalho apresenta 5 variações de algoritmos genéticos (GAs), uma das heurísticas mais populares no campo de index tracking [2], [3], [9], e introduz uma comparação entre as soluções por elas apresentadas e o pacote IBM ILOG CPLEX, solver exato comumente utilizado para a solução de problemas de otimização.

Portanto, foi realizada uma análise a partir dos dados de experimentos computacionais de forma a responder a primeira pergunta central desta pesquisa: "O algoritmo genético se mostra como uma abordagem competitiva quando aplicado à um mercado incipiente e volátil como o mercado acionário brasileiro?". Outra perguta é: "algoritmos híbridos, que são aqueles que usam um solver exato na alocação de capital, têm desempenho melhor que os puros?".

A contribuição deste trabalho para a literatura se dá pela aplicação de dois modelos de otimização ao problema de index tracking, bem como uma comparação de 5 variações de algoritmos genéticos, puros e híbridos, em uma série de dados recente do índice de ações brasileiro IBOVESPA, benchmark ainda pouco estudado, mas de extrema importância para o mercado financeiro nacional. Os resultados mostram uma abordagem híbrida extremamente competitiva, retornando soluções com uma discrepância do solver geral menor do que 9\% nas situações de rebalanceamento único, e chegando a superar o CPLEX durante os testes com rebalanceamento. 
O restante deste trabalho está organizado da seguinte forma: Seção II mostra uma breve revisão da literatura sobre trabalhos relacionados, Seção III apresenta os modelos e heurísticas utilizadas, Seção IV apresenta o conjunto de dados utilizados, bem como as métricas usadas na análise, Seção V apresenta os resultados e discussões e a Seção $\mathrm{VI}$ apresenta as conclusões.

\section{TRABALhOS RELACIONADOS}

Durante a evolução dos estudos sobre problemas de index tracking, diferentes modelos foram propostos, com diferentes definições do tracking error [1] $-[4]$, inclusões de restrições como UCITS, diretivas para operação de fundos de investimento na União Europeia [7], [10], ou restrições de limites de participação de cada ativo, de forma a garantir a diversificação do portfólio.

As metaheurísticas mostram-se como uma alternativa viável para lidar com o aumento de tempo necessitado por um solver exato devido ao aumento da complexidade do problema ao adicionar essas restrições. Uma revisão da literatura mostra que algoritmos evolucionários (nos quais estão incluídos algoritmos genéticos) são uma das principais estratégias para a otimização de portfólio [2], [5]-[7], [9], [11]-[13]. Esses algoritmos se mostram essenciais para portfólios com restrição de cardinalidade, problema de natureza combinatorial e com complexidade crescente à medida em que a quantidade de ativos presente no índice a ser perseguido aumenta.

Diversos métodos heurísticos foram propostos, como os métodos de best-extension-by-one [14], kernel search [6], particle swarm optimzation [15], [16], ou os algoritmos genéticos [2], [3], [5], [17]. Todos esses métodos permitiram a obtenção de boas soluções em tempos consideravelmente menores quando comparados com um método exato de solução. Proposto por John Holland em 1995 [18], o algoritmo genético é um dos métodos evolucionários mais antigos existentes, contudo, continua a ser um dos mais populares no campo de index tracking.

$\mathrm{Na}$ aplicação dos algoritmos genéticos para a seleção de portfólio, uma das abordagens que se mostra mais efetiva é a combinação do GA com o solver exato. Desta forma, o algoritmo é responsável pela parte combinatorial do problema, gerando um conjunto inicial de possíveis soluções, chamado de universo, enquanto o solver exato é responsável pela otimização do modelo simplificado, considerando apenas os dados dos ativos presentas na possível solução. Esta abordagem híbrida garante ao solver agilidade na otimização da possível solução, enquanto o GA é responsável por eliminar os piores candidatos e substitui-los por outros melhores, garantindo um avanço na busca da solução à cada iteração. O resultado é um algoritmo capaz de retornar resultados competitivos em tempos menores do que uma solução ótima obtida com o solver exato [2], [3], [5], [6], [13].

Outra abordagem são os chamados GAs puros. Para esta estratégia, o algoritmo genético é também responsável por atribuir o peso de cada ativo na possível solução com a utlização de representações, mutações e crossover específicos, [17], [19]-[23]. Considerando essas possibilidades, faz-se necessária uma extensa análise a partir de experimentos computacionais para testar a versatilidade e eficiência de cada heurística aplicada a diferentes modelos e conjuntos de dados, especialmente em momentos de alta volatilidade [8].

Este trabalho apresenta uma comparação entre os já populares algoritmos genéticos, aplicados pela primeira vez em um conjunto de dados recente sobre o índice de ações brasileiro, de forma a analisar a eficiência dos algoritmos para a solução de um modelo linear e um modelo quadrático.

\section{Abordagem Evolucionária PARA A SELEÇÃo DE PORTFÓLIO}

\section{A. Modelos}

Neste trabalho foram analisados dois modelos de otimização, um linear, presente em [3], e outro quadrático, presente em [2]. Foram aplicadas as mesmas restrições à ambos os modelos: limites de participação para cada ativo e a restrição de cardinalidade.

Seja o benchmark considerado formado por $N$ ativos durante um período $\{1,2, \ldots, T\}$ e tenha os retornos representados pela série $\left\{R^{1}, R^{2}, \ldots, R^{T}\right\}$. O retorno de um ativo $i$ componente do índice é dado pela série $\left\{r_{i}^{1}, r_{i}^{2}, \ldots, r_{i}^{T}\right\} ; i=\{1, \ldots, N\}$. O investidor deve manter o portfólio $P=\left\{x_{1}, x_{2}, \ldots, x_{N}\right\}$ composto por $K$ ativos, onde $x_{i}$ é o peso do ativo $i$ no portfólio, não sendo permitidas vendas à descoberto. $\mathrm{O}$ retorno do portfólio no tempo $t$ é dado por $\sum_{i=1}^{N} r_{i}^{t} \cdot x_{i}$.

Os limites de participação definem níveis máximos e mínimos de um ativo que faça parte da solução, garantindo um nível de diversificação ao portfólio. A variável binária $z_{i}$ assume valor 0 se o ativo $i$ não faz parte da solução, e 1 em caso contrário.

A definição de tracking error utilizada no modelo linear é o desvio médio absoluto (MAD), sendo a média dos valores absolutos das diferenças entre os retornos do portfólio e do índice.

$$
M A D=\frac{1}{T} \sum_{t=1}^{T}\left|R^{t}-\sum_{i=1}^{N} r_{i}^{t} x_{i}\right|
$$

Para o modelo quadrático, a definição utilizada foi o desvio médio quadrático (MSE), sendo a média dos quadrados das diferenças dos retornos.

$$
M S E=\frac{1}{T} \sum_{t=1}^{T}\left(R^{t}-\sum_{i=1}^{N} r_{i}^{t} x_{i}\right)^{2}
$$

A formulação dos dois modelos foi feita como problemas de programação inteira mista, onde $z_{i}$ são variáveis binárias que podem assumir valor 0 (traduz-se como ativo não incluso na solução) ou 1 (ativo incluso), da mesma forma que em Wang et al. [3] e Torrubiano e Suárez [2].

\section{B. Modelo Linear}

$$
\underset{\mathrm{x}, q^{+}, q^{-}, \mathrm{z}}{\operatorname{Min}} \frac{1}{T} \sum_{t=1}^{T}\left(q_{t}^{+}+q_{t}^{-}\right)
$$




$$
\begin{array}{ll}
\text { s.a. } & q_{t}^{+}-q_{t}^{-}=R^{t}-\sum_{i=1}^{N} r_{i}^{t} x_{i}, \quad t=1,2, \ldots, T \\
& \sum_{i=1}^{N} z_{i}=K \\
& \\
L_{i} z_{i} \leq x_{i} \leq U_{i} z_{i} & i=1,2, \ldots, N \\
\sum_{i=1}^{N} x_{i}=1 & \\
z_{i} \in\{0,1\}, & i=1,2, \ldots, N \\
q_{t}^{+} \geq 0, & t=1,2, \ldots, T
\end{array}
$$

As variáveis $q_{t}^{+}$e $q_{t}^{-}$e a equação $3 \mathrm{~b}$ são parte do ajuste feito na função objetivo (MAD), visto que a definição original é linear por partes e não diferenciável. A equação (3c) é a restrição de cardinalidade, a qual garante que a solução terá exatamente $K$ ativos, definido como 10 ativos para ambos os modelos. As restrições (3d) são os limites de participação. Neste trabalho, foram definidos $L_{i}=0.01, U_{i}=0.5, \quad i=$ $1,2, \ldots, N$. Por fim, a restrição (3e garante a proporção do portfólio, isto é, não haverá nenhum percentual alocado em caixa, tampouco alavancagem.

\section{Modelo Quadrático}

$$
\operatorname{Min}_{w}\left[\frac{1}{2} \boldsymbol{w}^{\prime} \cdot \mathbf{H} \cdot \boldsymbol{w}-\boldsymbol{g}^{\prime} \cdot \boldsymbol{w}\right]
$$

onde $\boldsymbol{H}$ e $\boldsymbol{g}$ são matrizes com as seguintes definições

$$
\begin{gathered}
H_{i j}=\frac{1}{T} \sum_{t=1}^{T} r_{i}^{t} r_{j}^{t} \\
g_{i}=\frac{1}{T} \sum_{t=1}^{T} r_{i}^{t} R^{t} \\
\text { s.a. } \quad \sum_{i=1}^{N} x_{i}=1 \\
\sum_{i=1}^{N} z_{i}=K \\
L_{i} z_{i} \leq x_{i} \leq U_{i} z_{i} \quad i=1,2, \ldots, N \\
w_{i} \geq 0, \forall i=1, \ldots, N \\
z_{i} \in\{0,1\}
\end{gathered}
$$

As matrizes $\mathbf{H}$ e $\boldsymbol{g}$ representam o problema quadrático em sua forma matricial. As equações (4d) (4f), (4g) e (4e) se referem, respectivamente, à alocação do capital, limites de participação, proibição de vendas à descoberto e cardinalidade, w é o vetor coluna com os pesos de cada ativo na solução.

\section{Algoritmos}

Dois tipos de algoritmos genéticos foram usados nos experimentos: um GA puro baseado em um processo de mutação gaussiana e em um torneio binário para a seleção do melhor indivíduo, e uma abordagem híbrida. Foram comparadas quarto variações do GA híbrido, com duas variações de mutações e dois algoritmos de crossover.

Os algoritmos de crossover aplicados foram: 1-point order crossover usada por Sant'Anna [5], e a rotina Random Assorting Recombination (RAR), que consiste em separar em conjuntos os ativos presentes nos dois, em cada um e em nenhum dos pais. Uma melhor descrição do método RAR pode ser encontrada em seu artigo original [2], [13]. Para cada crossover, foram usados dois processos de mutação: simples e duplo, que consistem em trocar aleatoriamente um (simples) ou dois (duplo) ativo(s) de uma possível solução por outro(s) não incluso(s). o pseudocódigo geral utilizado para os GAs foi:

1) Criação de uma população inicial com $P$ possíveis soluções aleatórias.

2) Avaliar o valor da função objetivo de todos os indivíduos.

3) Até tempo $\leq$ limite :

a) Escolher aleatoriamente dois pais.

b) Realizar o crossover, gerando duas novas possíveis soluções (crianças).

c) $85 \%$ de chance de ocorrência de mutação nas crianças.

d) Inserir as crianças na população.

e) Retirar os dois piores pais da nova população.

A diferença entre os GAs híbridos está na rotina aplicada nos passos 3.b e 3.c. Cada algoritmo foi referido durante este trabalho de acordo com o artigo em que foi apresentado, seguido de uma referência ao tipo de mutação aplicado:

O GA puro utiliza uma representação (genótipo) com um vetor de duas dimensões: a primeira equivale à um vetor linha binário com os ativos inclusos na solução, por exemplo, o vetor $[0,1,1,0]$ inclui apenas o segundo e terceiro ativo. A segunda dimensão contém o peso de cada ativo na solução. Seguindo o exemplo anterior, se o segundo ativo compõe $40 \%$ do portfólio, enquanto o terceiro $60 \%$, a segunda dimensão do vetor seria $[0,0.4,0.6,0]$. Neste algoritmo, a atribuição dos pesos é igualmente distribuída no início, e a cada mutação, um novo peso $w_{i}^{\prime}$ é atribuído ao ativo $i$, onde $w_{i}^{\prime}$ é um número Gaussiano gerado de forma aleatória, com o centro da distribuição sendo o antigo peso, e respeitando os limites de cardinalidade $w_{i}^{\prime}=\max \left(\min \left(N\left(w_{i}, \sigma\right), U\right), L\right)$, diferentemente dos algoritmos híbridos, nos quais o solver CPLEX é responsável por esta atribuição. Após a mutação, é realizada uma normalização dos pesos, de modo à atender a restrição da alocação de capital (3e), para isso, são atribuídos novos pesos $w_{i}^{\prime \prime}=w_{i}^{\prime} / \sum w_{i}^{\prime}$ [20], [24]. O crossover utilizado nesse GA foi o 1-point order.

Em resumo, foram analisadas 5 variações de algoritmos genéticos:

- GA-Sant'Anna simples [5]

Crossover: 1-point order [5]

Mutação: Simples [5] 
- GA-Sant'Anna dupla [5]

Crossover: 1-point order [5]

Mutação: Dupla [5]

- GA-Torrubiano simples [2]

Crossover: RAR [2]

Mutação: Simples [5]

- GA-Torrubiano dupla [2]

Crossover: RAR [2]

Mutação: Dupla [5]

\section{- GA-Puro}

Crossover: 1-point order [5]

Mutação: Gaussiana [20]

\section{EXPERIMENTOS}

\section{A. Dados}

O dataset utilizado corresponde aos preços de fechamento dos ativos que compuseram o índice do mercado brasileiro (Ibovespa) de 31-07-2016 até 28-12-2018, excluindo dados inconsistentes de ações que deixaram de existir ou sofreram processos de M\&As neste período. Este conjunto contém informação sobre 86 ativos, com séries de retornos destes ativos durante 589 dias, contendo períodos de crise política e a efetivação de um processo de impeachment e alta volatilidade do mercado,

O período de in-sample utilizado foi de 393 dias. Esse período foi intencionalmente maior, de modo a tentar obter soluções com melhor qualidade [25]. O solver IBM ILOG CPLEX V 12.10.0.3 foi configurado para rodar por um tempo limite de 60 minutos, enquanto o limite para o algoritmo genético foi configurado em 5 minutos para cada instância.

Ambos os modelos foram solucionados em uma estratégia sem rebalancemanto, inicialmente pelo solver completo. Em seguida foram resolvidos 30 vezes por cada GA, e as soluções foram comparadas. Após isso, os dois modelos foram resolvidos pelo CPLEX e pelo melhor GA da etapa anterior, mas para a estratégia de rebalanceamento de portfólio mensal, trimestral e semestral. Esta abordagem permite aos modelos lidar com dados mais recentes a medida em que novas otimizações são feitas.

Para a primeira etapa sem rebalancemento, o objetivo principal foi determinar qual dos GAs performou melhor em qual modelo, retornando soluções mais próximas do benchmark e compará-las as soluções retornadas pelo CPLEX em cada modelo. Desta forma, buscou-se analisar se o algoritmo retorna ou não soluções competitivas. Para isso, foram analisados os retornos cumulativos, gaps máximos, médios e mínimos entre o CPLEX e o GA em termos de função objetivo, e o número médio de iterações realizadas por cada algoritmo, de forma a mensurar a eficiência de cada algoritmo.

$$
G a p=\frac{O b j_{G A}-O b j_{C P L E X}}{O b j_{C P L E X}}
$$

O retorno cumulativo $C_{i}$ no tempo $i, \operatorname{com} r_{i}$ sendo o retorno do portfólio, é calculado como

$$
C_{i}=C_{i-1} \cdot\left(1+r_{i}\right) \quad C_{0}=1 ; i=\{1, \ldots, T\}
$$

$\mathrm{Na}$ segunda etapa, o objetivo foi analisar a performance do algoritmo genético escolhido quando aplicado à cada modelo no ambiente de rebalanceamento, e monitorar o trade-off entre tempo computacional e qualidade da solução obtida pelo algoritmo quando comparado com o CPLEX. Para isso, o CPLEX rodou com o tempo limite de cada otimização configurado para 30 minutos, enquanto o GA vencedor rodou apenas uma vez por 5 minutos. Foram analisados os retornos cumulativos, os gaps entre os tracking errors durante todo o período out-of-sample, comparando o total no período, o máximo, mínimo, e o desvio padrão. Por fim, foi analisado também o turnover mensal médio de cada portfólio, visto que, apesar de potencialmente retornar melhores resultados, a troca de ativos do portfólio incorre em custos de transação que podem inviabilizar a estratégia.

\section{Resultados e Discuss Ão}

\section{A. Otimização em Período Único}

As tabelas $\Pi \mathrm{e} \Pi$ IIresumem os resultados obtidos para os GAs aplicados nos modelos linear e quadrático, respectivamente. A primeira linha "MAD" / "MSE" apresenta o melhor valor da função objetivo obtido por cada GA durante o período insample enquanto o valor do CPLEX está entre parênteses, a segunda linha mostra a quantidade média de iterações de cada GA. A linha "Gap Médio" apresenta a média dos gaps obtidos entre as 30 instâncias de cada GA, enquanto "Gap Máx" / "Gap Mín" indicam respectivamente os gaps máximos e mínimos dentre as 30 instâncias. Por fim, a última linha indica o desvio padrão entre os 30 gaps de cada algoritmo.

O GA-puro obteve a pior performance em ambos os modelos, superando em mais de 4 vezes o segundo maior número de iterações no modelo linear, obtido pelo GA-Torrubiano duplo, obteve também o pior valor de função objetivo.

O GA-Sant'Anna simples obteve consistentemente a melhor performance em ambos os modelos, obtendo o menor gap médio, e seu gap máximo foi ainda o menor para o modelo linear, e o segundo menor para o modelo quadrático. Além disto obteve o menor desvio padrão, mostrando que este GA apresentou uma menor variação entre suas soluções, mostrando-se mais confiável e repetitivo. Vale ainda notar que ambos os GA-Sant'Anna foram capazes de encontrar a mesma solução do CPLEX.

Em uma análise geral, os algoritmos genéticos híbridos se mostraram extremamente competitivos, obtendo soluções com um erro menor do que $6 \%$ para o modelo linear e $9 \%$ para o modelo quadrático, em apenas $1 / 12$ do tempo necessário pelo CPLEX. Isto mostra que os GAs híbridos podem ser alternativas viáveis para ajudar na tomada de decisão de um gestor.

A partir dessa análise, o GA-Sant'Anna simples foi escolhido para a etapa de rebalanceamentos do portfólio. Desta 
forma, a solução do GA foi comparada com a solução do CPLEX nas três diferentes janelas de rebalanceamento (mensal, trimestral e semestral).

TABLE I

COMPARAÇÃO DOS GAS PARA O MODELO LINEAR

\begin{tabular}{|c|c|c|c|c|c|}
\hline GA & Puro & $\begin{array}{c}\text { Sant- } \\
\text { Simples }\end{array}$ & $\begin{array}{c}\text { Sant- } \\
\text { Duplo }\end{array}$ & $\begin{array}{c}\text { Torr- } \\
\text { Simples }\end{array}$ & $\begin{array}{c}\text { Torr- } \\
\text { Duplo }\end{array}$ \\
\hline $\begin{array}{c}\text { MAD } \\
(1.64 \mathrm{e}-3)\end{array}$ & $1.95 \mathrm{e}-3$ & $1.64 \mathrm{e}-3$ & $1.64 \mathrm{e}-3$ & $1.80 \mathrm{e}-3$ & $1.77 \mathrm{e}-3$ \\
\hline Iterações & 6775 & 670 & 723 & 1588 & 1580 \\
\hline Gap Máx & $1.32 \mathrm{e}+0$ & $6.40 \mathrm{e}-2$ & $1.76 \mathrm{e}-1$ & $3.02 \mathrm{e}-1$ & $3.22 \mathrm{e}-1$ \\
\hline Gap Mín & $1.90 \mathrm{e}-1$ & $0.00 \mathrm{e}+0$ & $0.00 \mathrm{e}+0$ & $1.00 \mathrm{e}-1$ & $8.06 \mathrm{e}-2$ \\
\hline Std dev & $2.69 \mathrm{e}-1$ & $2.11 \mathrm{e}-2$ & $3.69 \mathrm{e}-2$ & $3.84 \mathrm{e}-2$ & $6.30 \mathrm{e}-2$ \\
\hline
\end{tabular}

TABLE II

COMPARAÇÃo DOS GAS PARA O MODELO QUADRÁTICO

\begin{tabular}{|c|c|c|c|c|c|}
\hline GA & Puro & $\begin{array}{c}\text { Sant- } \\
\text { Simples }\end{array}$ & $\begin{array}{c}\text { Sant- } \\
\text { Duplo }\end{array}$ & $\begin{array}{c}\text { Torr- } \\
\text { Simples }\end{array}$ & $\begin{array}{c}\text { Torr- } \\
\text { Duplo }\end{array}$ \\
\hline $\begin{array}{c}\text { Best MSE } \\
(4.92 \mathrm{e}-6)\end{array}$ & $7.09 \mathrm{e}-6$ & $4.92 \mathrm{e}-6$ & $4.92 \mathrm{e}-6$ & $4.92 \mathrm{e}-6$ & $5.01 \mathrm{e}-6$ \\
\hline Iterações & 13170 & 1900 & 2971 & 7634 & 8102 \\
\hline Gap Médio & $1.47 \mathrm{e}+0$ & $3.12 \mathrm{e}-3$ & $8.41 \mathrm{e}-3$ & $1.57 \mathrm{e}-2$ & $8.86 \mathrm{e}-2$ \\
\hline Gap Máx & 4.99 & $1.25 \mathrm{e}-2$ & $3.44 \mathrm{e}-2$ & $1.12 \mathrm{e}-1$ & $1.75 \mathrm{e}-1$ \\
\hline Gap Mín & $4.41 \mathrm{e}-1$ & $0.00 \mathrm{e}+0$ & $0.00 \mathrm{e}+0$ & $0.00 \mathrm{e}+0$ & $1.79 \mathrm{e}-2$ \\
\hline Std Dev & $8.59 \mathrm{e}-1$ & $4.45 \mathrm{e}-3$ & $7.55 \mathrm{e}-3$ & $2.40 \mathrm{e}-2$ & $3.74 \mathrm{e}-2$ \\
\hline
\end{tabular}

Em uma breve comparação entre as soluções retornadas por cada modelo, nenhum dos dois se mostrou claramente melhor que o outro. Apesar de ter apresentado menores tracking errors durante o período out-of-sample, o modelo linear teve um retorno cumulativo pouco menor do que o modelo quadrático. Ademais, a performance dos dois modelos durante o período foi semelhante, como mostrado na tabela III

Fig. 1. Performance dos modelos linear e quadrático

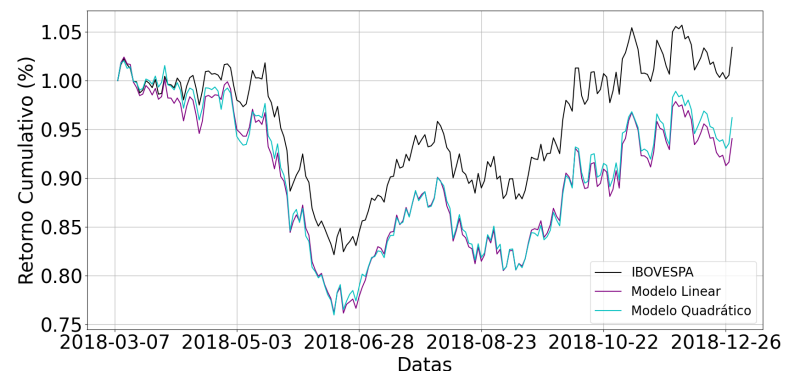

TABLE III

Retorno Cumulativo E OBJetivos no OUT-OF-SAMPLE

\begin{tabular}{|c|c|c|c|}
\hline Modelo & $\begin{array}{c}\text { Retorno Cum } \\
(1.03 \mathrm{e} 0)\end{array}$ & MSE & MAD \\
\hline Lin & $94.06 \mathrm{e}-2$ & $1.15 \mathrm{e}-5$ & $2.51 \mathrm{e}-3$ \\
\hline Quad & $96.21 \mathrm{e}-2$ & $1.49 \mathrm{e}-5$ & $2.73 \mathrm{e}-3$ \\
\hline
\end{tabular}

\section{B. Otimização com Rebalanceamentos}

O GA-Sant'Anna Simples foi aplicado na solução dos modelos linear e quadrático em uma estratégia de rebalanceamento periódico do portfólio. Os resultados dos experimentos com rebalanceamento estão mostrados nas tabelas IV e V A primeira e segunda linhas mostram, respectivamente, os retornos cumulativos obtidos pelo GA e CPLEX. A terceira linha indica qual foi o gap entre a função objetivo do algoritmo e do solver durante todo o período out-of-sample. as linhas "Gap Máx" e "Gap Mín" indicam os gaps máximos e mínimos de cada modelo durante o período, e as duas últimas linhas indicam o turnover de cada portfólio.

Para o modelo linear, o GA apresentou-se como uma melhor opção em todas as janelas de rebalanceamento, com um retorno cumulativo muito mais próximo do índice nos rebalanceamentos trimestral e semestral, e pouco abaixo do retorno obtido pelo solver para a janela mensal. O tracking error total ao longo do período foi menor para o GA nas janelas mensal e trimestral, enquanto no rebalanceamento semestral, o GA teve um valor de função objetivo maior, mas novamente não superando $9 \%$. Além disso, o desvio padrão entre os gaps do GA e do CPLEX foram extremamente altos, o que indica que o GA e CPLEX obtiveram soluções com performances muito diferentes. A superioridade do GA está melhor representada nas figuras $2,3 \mathrm{e} 4$.

Fig. 2. GA vs CPLEX mensal no modelo quadrático

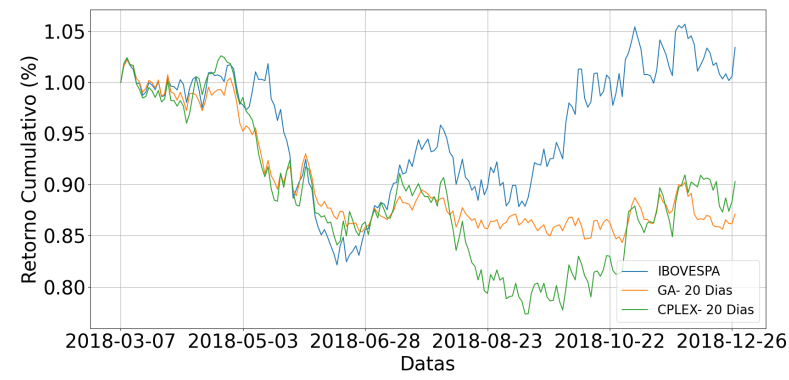

Fig. 3. GA vs CPLEX trimestral no modelo quadrático

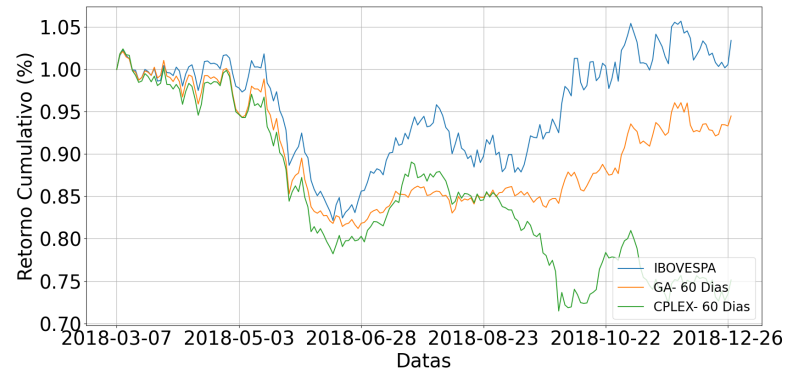

Para o modelo quadrático, o GA mostrou-se novamente como uma alternativa competitiva ao solver. Os retornos cumulativos foram mais próximos do Ibovespa no GA em 
Fig. 4. GA vs CPLEX semestral no modelo quadrático

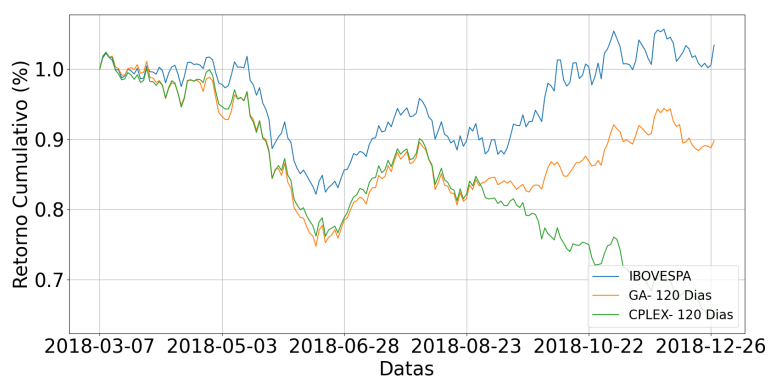

TABLE IV

COMPARAÇÃO GA-SANT-SIMPLES PARA ESTRATÉGIA DE REBALANCEAMENTO NO MODELO LINEAR

\begin{tabular}{|c|c|c|c|}
\hline Rebalanceamento & 20 Dias & 60 Dias & 120 Dias \\
\hline R. Cum GA & 0.87 & 0.94 & 0.89 \\
\hline R. Cum CPLEX & 0.90 & 0.75 & 0.65 \\
\hline Gap Total MSA & $-2.55 \mathrm{e}-2$ & $-1.09 \mathrm{e}-1$ & $8.32 \mathrm{e}-2$ \\
\hline Gap Máx MSA & $1.19 \mathrm{e}+2$ & $2.85 \mathrm{e}+1$ & $1.28 \mathrm{e}+2$ \\
\hline Gap Mín MSA & $-1.00 \mathrm{e} 0$ & $-9.89 \mathrm{e}-1$ & $-9.70 \mathrm{e}-1$ \\
\hline Gaps Std Dev & $1.06 \mathrm{e}+1$ & $3.28 \mathrm{e}+0$ & $9.93 \mathrm{e}+0$ \\
\hline Turnover GA & $4.38 \mathrm{e}+0$ & $6.20 \mathrm{e}-1$ & $1.67 \mathrm{e}-1$ \\
\hline Turnover CPLEX & $8.21 \mathrm{e}+0$ & $9.00 \mathrm{e}-1$ & $1.46 \mathrm{e}-1$ \\
\hline
\end{tabular}

todas as janelas de rebalanceamento. Já o tracking error do período foi menor no CPLEX do que no algoritmo genético, conforme mostrado nas figuras $5,6 \mathrm{e} 7$

Fig. 5. GA VS CPLEX mensal no modelo quadrático

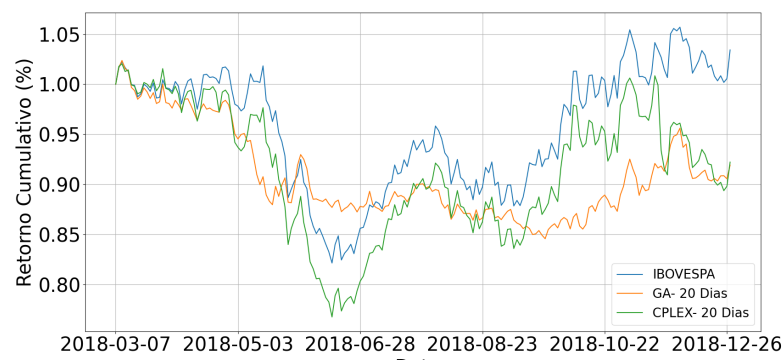

Datas

Em ambos os modelos o turnover foi, como esperado, decrescente conforme a janela de rebalanceamento aumentou. Este resultado é intuitivo, visto que, à medida em que a janela de rebalanceamento aumenta, menos rebalanceamentos serão feitos, portanto a rotatividade dos ativos na carteira diminuirá.

Para comparar as estratégias de investimento, as figuras 8 e 9. Quando comparando as estratégias de rebalanceamento com a otimização única, não há um vencedor claro. É intuitivo imaginar que, como um rebalanceamento mensal insere dados mais recentes nos modelos, a solução desta estratégia teria melhor performance quando comparada aos demais, entretanto
Fig. 6. GA VS CPLEX trimestral no modelo quadrático

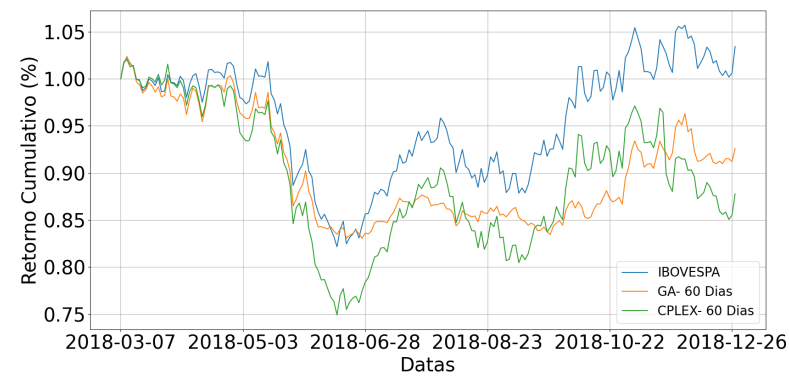

Fig. 7. GA VS CPLEX semestral no modelo quadrático

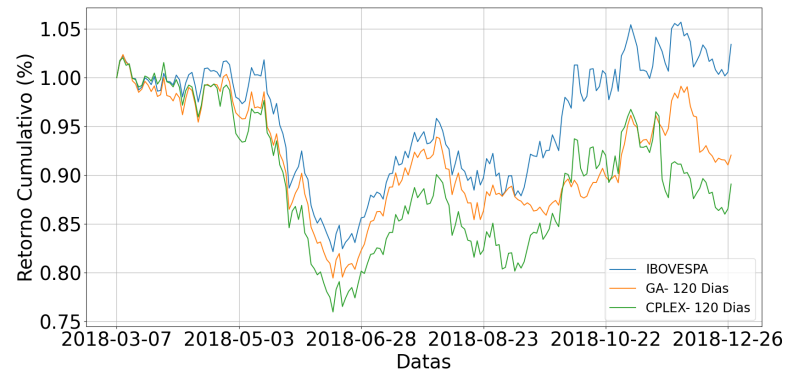

TABLE V

COMPARAÇão GA-SANT-SimPLES PARA ESTRATÉGIA DE REBALANCEAMENTO NO MODELO QUADRÁTICO

\begin{tabular}{|c|c|c|c|}
\hline Rebalanceamento & 20 Dias & 60 Dias & 120 Dias \\
\hline R. Cum GA & 0.92 & 0.92 & 0.92 \\
\hline R. CUm CPLEX & 0.92 & 0.88 & 0.89 \\
\hline Gap Total MSE & $2.67 \mathrm{e}+0$ & $1.36 \mathrm{e}+0$ & $1.03 \mathrm{e}+0$ \\
\hline Gap Máx MSE & $3.01 \mathrm{e}+5$ & $2.91 \mathrm{e}+5$ & $2.91 \mathrm{e}+5$ \\
\hline Gap Mín MSE & $-1.03 \mathrm{e}+0$ & $-0.97 \mathrm{e}+0$ & $-4.12 \mathrm{e}+0$ \\
\hline Gaps Std Dev & $2.25 \mathrm{e}+4$ & $2.10 \mathrm{e}+4$ & $2.19 \mathrm{e}+4$ \\
\hline Turnover GA & $4.18 \mathrm{e}+0$ & $5.90 \mathrm{e}-1$ & $1.37 \mathrm{e}-1$ \\
\hline Turnover CPLEX & $2.85 \mathrm{e}+0$ & $4.42 \mathrm{e}-1$ & $9.80 \mathrm{e}-2$ \\
\hline
\end{tabular}

isto não ocorre, e a este rebalanceamento mensal chega, em alguns períodos, a ser pior do que as demais soluções, esta situação pode ainda se agravar ao considerar taxas de transação, que não foram contabilizadas neste estudo.

\section{Comparações Estatísticas do Ibovespa}

Como forma de analisar a volatilidade e o risco do mercado, são apresentados na tabela VI os dados estatísticos da série de retornos do índice Ibovespa durante os anos de 2016, 2017 e 2018. Os períodos correspondem do dia 02 de Janeiro até o último dia útil de cada ano. $\mathrm{O}$ ano de 2018 obteve a média de retornos mais baixa, enquanto 2016 obteve a melhor média de retornos. Durante o ano de 2016, o retorno médio foi de $0.17 \%$, com um desvio padrão consideravelmente alto, assim como uma distorção igualmente alta e positiva, indicando uma calda 
Fig. 8. Rebalanceamento VS otimização única no modelo linear

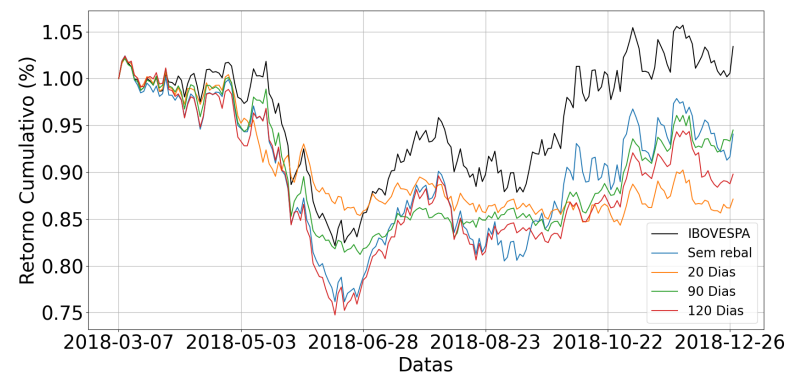

Fig. 9. Rebalanceamento VS otimização única no modelo quadrático

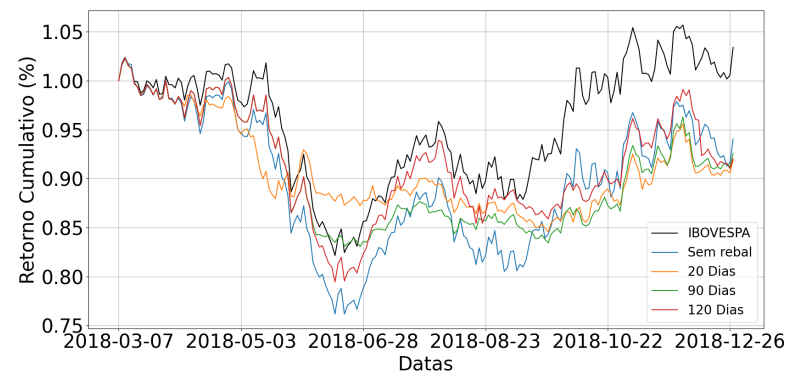

maior à direita da média dos retornos, e a curtose igualmente alta, indicando uma distribuição espaçada. Estes fatos indicam um mercado assimétrico, com mais retornos acima da média.

$\mathrm{O}$ ano de 2017 obteve a maior distorção dentre todos os anos, enquanto uma curtose extremamente alta, indicando retornos espaçados de forma extremamente assimétrica, com valores à esquerda da média, estes fatores indicam uma alta volatilidade no mercado.

Por fim, o ano de 2018 apresentou a pior média de retornos, mas desta vez com a menor distorção, apesar de negativa, e menor curtose, o que indica uma distribuição de retornos mais próxima da normal, e consideravelmente simétrica. Estes fatos demonstram uma menor volatilidade durante o ano de 2018, contudo, o mesmo corresponde ao período de menores retornos.

TABLE VI

ESTATÍSTICAS ANUAIS DOS RETORNOS DO IBOVESPA

\begin{tabular}{|c|c|c|c|}
\hline & 2016 & 2017 & 2018 \\
\hline Amostra & 245 & 246 & 249 \\
\hline Média & $1.74 \mathrm{e}-3$ & $1.09 \mathrm{e}-3$ & $6.70 \mathrm{e}-4$ \\
\hline Std Dev & $1.67 \mathrm{e}-2$ & $1.20 \mathrm{e}-2$ & $1.40 \mathrm{e}-2$ \\
\hline Distorção & $1.63 \mathrm{e}-1$ & $-1.59 \mathrm{e}+0$ & $-7.50 \mathrm{e}-2$ \\
\hline Curtose & $1.07 \mathrm{e}+0$ & $1.19 \mathrm{e}+1$ & $4.43 \mathrm{e}-1$ \\
\hline
\end{tabular}

\section{CONCLUSÕES}

Este estudo apresentou uma comparação entre a aplicação de 5 GAs em dois modelos de otimização aplicados a um conjunto de dados do mercado brasileiro incluindo um período de alta volatilidade no mercado, contendo dados durante o período de impeachment e da maior greve do país nos últimos 20 anos, no que ficou conhecida como greve dos caminhoneiros.

O algoritmo genético puro mostrou limitações quanto à sua aplicação para o problema de index tracking apresentado. Entretanto, a abordagem híbrida resultou em um algoritmo extremamento competitivo, tanto na estratégia de otimização única, quanto nos rebalanceamentos periódicos. A abordagem híbrida do GA-Sant'Anna simples foi capaz de superar o CPLEX para a estratégia de rebalanceamento, ambiente em que possui ainda mais valor, visto que cada otimização foi realizada em 5 minutos, ao invés dos 30 minutos do solver completo, isto possibilita uma economia de 250 minutos para otimizações mensais, por exemplo. Para uma otimização única, o GA foi novamente capaz de entregar soluções competitivas, igualando o CPLEX, e entregando soluções no máximo $9 \%$ piores do que o solver exato. A partir desses resultados, esta abordagem híbrida mostra-se de útil aplicação principalmente em situações como a criação ou manutenção de fundos negociados passivos em bolsa, chamados ETFs ( $E x$ change Traded Funds), especialmente em momentos de alta volatilidade do mercado, no qual o gestor pode desejar fazer diversas simulações antes da tomada de decisão.

Quando comparadas as estratégias de rebalanceamento com uma simples otimização, não há uma dominância clara. Rebalancear o portfólio mensalmente não trouxe o retorno cumulativo mais próximo do índice, tampouco o menor tracking error dentre as outras alternativas, mesmo não considerando o impacto dos custos de rotacionar os ativos na carteira de investimentos sobre o retorno.

Em resumo, o GA híbrido apresentou uma performance excelente e mostrou-se uma alternativa extremamente viável para a tomada de decisão da seleção de portfólio de forma rápida e confiável. Com respeito as estratégias de rebalanceamento, estudos futuros podem apresentar uma extensão deste trabalho na tentativa de melhor entender os fatores que impactam na qualidades dessa abordagem. Outra direção interessante a ser explorada pode ser o uso de ratings na modelagem [26]-[29]

\section{AGRADECIMENTOS}

Este trabalho foi parcialmente apoiado pela Capes e CNPq.

\section{REFERENCES}

[1] J. Beasley, N. Meade, and T. J. Chang, "An evolutionary heuristic for the index tracking problem," European Journal of Operational Research, vol. 148, pp. 621-643, 082003.

[2] R. Torrubiano and A. Suárez, "A hybrid optimization approach to index tracking," Annals OR, vol. 166, pp. 57-71, 022009.

[3] M. Wang, C. Xu, F. Xu, and H. Xue, "A mixed 0-1 lp for index tracking problem with cvar risk constraints," Annals of Operations Research, vol. 196, 072012.

[4] M. Rudolf, H.-J. Wolter, and H. Zimmermann, "A linear model for tracking error minimization," Journal of Banking \& Finance, vol. 23, no. 1, pp. 85 - 103, 1999. [Online]. Available: http: //www.sciencedirect.com/science/article/pii/S0378426698000764 
[5] L. Sant'Anna, T. Filomena, P. Guedes, and D. Borenstein, "Index tracking with controlled number of assets using a hybrid heuristic combining genetic algorithm and non-linear programming," Annals of Operations Research, vol. 258, 112017.

[6] G. Guastaroba and M. Speranza, "Kernel search: An application to the index tracking problem," European Journal of Operational Research, vol. 217, no. 1, pp. 54 - 68, 2012. [Online]. Available: http://www.sciencedirect.com/science/article/pii/S0377221711008071

[7] A. Scozzari, F. Tardella, S. Paterlini, and T. Krink, "Exact and heuristic approaches for the index tracking problem with ucits constraints," Annals of Operations Research, vol. 205, 052012.

[8] M. Kaucic, "Equity portfolio management with cardinality constraints and risk parity control using multi-objective particle swarm optimization," Computers \& Operations Research, vol. 109, pp. 300-316, 2019. [Online]. Available: https://www.sciencedirect.com/ science/article/pii/S0305054819301273

[9] A. Almeida-Filho, D. de Lima Silva, and L. Ferreira, "Financial modelling with multiple criteria decision making: A systematic literature review," Journal of the Operational Research Society, pp. 1-19, 062020.

[10] L. Shu, F. Shi, and G. Tian, "High-dimensional index tracking based on the adaptive elastic net," Quantitative Finance, vol. 20, no. 9, pp. 1513-1530, 2020. [Online]. Available: https://doi.org/10. 1080/14697688.2020.1737328

[11] G. Dueck and P. Winker, "New concepts and algorithms for portfolio choice," Applied Stochastic Models and Data Analysis, vol. 8, pp. 159178,1992

[12] M. Sousa Lobo, M. Fazel, and S. Boyd, "Portfolio optimization with linear and fixed transaction," Annals OR, vol. 152, pp. 341-365, 03 2007.

[13] R. Moral-Escudero, R. Torrubiano, and A. Suarez, "Selection of optimal investment portfolios with cardinality constraints," 012006 , pp. 2382 2388.

[14] P. Mutunge and D. Haugland, "Minimizing the tracking error of cardinality constrained portfolios," Computers \& Operations Research, vol. 90, 092017.

[15] J. Kennedy and R. Eberhart, "Particle swarm optimization," in Proceedings of ICNN'95 - International Conference on Neural Networks, vol. 4, Nov 1995, pp. 1942-1948 vol.4.

[16] H. R. Golmakani and M. Fazel, "Constrained portfolio selection using particle swarm optimization," Expert Systems with Applications, vol. 38, no. 7, pp. 8327 - 8335, 2011. [Online]. Available: http://www.sciencedirect.com/science/article/pii/S0957417411000406

[17] C.-C. Lin and Y.-T. Liu, "Genetic algorithms for portfolio selection problems with minimum transaction lots," European Journal of Operational Research, vol. 185, no. 1, pp. 393 - 404, 2008. [Online]. Available: http://www.sciencedirect.com/science/article/pii/S0377221707000057

[18] J. H. Holland, Adaptation in Natural and Artificial Systems: An Introductory Analysis with Applications to Biology, Control and Artificial Intelligence. Cambridge, MA, USA: MIT Press, 1992.

[19] K. Anagnostopoulos and G. Mamanis, "The mean-variance cardinality constrained portfolio optimization problem: An experimental evaluation of five multiobjective evolutionary algorithms," Expert Syst. Appl., vol. 38, pp. 14 208-14217, 052011.

[20] F. Streichert, H. Ulmer, and A. Zell, "Evaluating a hybrid encoding and three crossover operators on the constrained portfolio selection problem," vol. 1, 07 2004, pp. 932 - 939 Vol.1.

[21] S. K. Mishra, G. Panda, and R. Majhi, "A comparative performance assessment of a set of multiobjective algorithms for constrained portfolio assets selection," Swarm and Evolutionary Computation, vol. 16, pp. 38 - 51, 2014. [Online]. Available: http://www.sciencedirect.com/science/ article/pii/S2210650214000029

[22] F. Garcia, F. Guijarro, and J. Oliver, "Index tracking optimization with cardinality constraint: a performance comparison of genetic algorithms and tabu search heuristics," Neural Computing and Applications, vol. 30, 102018.

[23] C. Fonseca and P. Fleming, "Genetic algorithms for multiobjective optimization: Formulation discussion and generalization," the fifth Intl conference on Genetic Algorithms, vol. 93, 021999.

[24] K. Liagkouras and K. Metaxiotis, "Examining the effect of different configuration issues of the multiobjective evolutionary algorithms on the efficient frontier formulation for the constrained portfolio optimization problem," Journal of the Operational Research Society, vol. 69, no. 3, pp. 416-438, 2018. [Online]. Available: https: //doi.org/10.1057/jors.2016.38
[25] A. Gaivoronski, S. Krylov, and N. Wijst, "Optimal portfolio selection with benchmark tracking," European Journal of Operational Research, vol. 163, pp. 115-131, 052005.

[26] J. C. S. Silva, D. F. de Lima Silva, L. Ferreira, and A. T. de Almeida-Filho, "A dominance-based rough set approach applied to evaluate the credit risk of sovereign bonds," 4OR, Jan. 2021. [Online]. Available: https://doi.org/10.1007/s10288-020-00471-w

[27] D. F. de Lima Silva, L. Ferreira, and A. T. de Almeida-Filho, "A new preference disaggregation topsis approach applied to sort corporate bonds based on financial statements and expert's assessment," Expert Systems with Applications, vol. 152, p. 113369, 2020. [Online]. Available: https://www.sciencedirect.com/science/article/pii/ S0957417420301949

[28] D. F. de Lima Silva and A. T. de Almeida Filho, "Sorting with topsis through boundary and characteristic profiles," Computers \& Industrial Engineering, vol. 141, p. 106328, 2020. [Online]. Available: https://www.sciencedirect.com/science/article/pii/S0360835220300620

[29] D. F. de Lima Silva, J. C. S. Silva, L. G. O. Silva, L. Ferreira, and A. T. de Almeida-Filho, "Sovereign credit risk assessment with multiple criteria using an outranking method," Mathematical Problems in Engineering, vol. 2018, pp. 1-11, Sep. 2018. [Online]. Available: https://doi.org/10.1155/2018/8564764 